\title{
Microscopic Understanding of the Ionic Networks of "Water-in-Salt" Electrolytes
}

\author{
Xinyi Liu, ${ }^{1}$ Zhou Yu $\mathbb{D}^{2,3}$ Erik Sarnello ${ }^{2},{ }^{1}$ Kun Qian ${ }^{1 D},{ }^{1}$ Soenke Seifert, ${ }^{4}$ \\ Randall E. Winans $\mathbb{D}^{3,4}$ Lei Cheng $\mathbb{B D}^{2,3}$ and Tao $\mathrm{Li} \mathbb{D}^{1,3,4}$ \\ ${ }^{1}$ Department of Chemistry and Biochemistry, Northern Illinois University, USA \\ ${ }^{2}$ Materials Science Division, Argonne National Laboratory, USA \\ ${ }^{3}$ Joint Center for Energy Storage Research, Argonne National Laboratory, USA \\ ${ }^{4} X$-Ray Science Division, Argonne National Laboratory, USA
}

Correspondence should be addressed to Lei Cheng; leicheng@anl.gov and Tao Li; taoli@anl.gov

Received 9 November 2020; Accepted 29 December 2020; Published 28 January 2021

Copyright ( 2021 Xinyi Liu et al. Exclusive Licensee Beijing Institute of Technology Press. Distributed under a Creative Commons Attribution License (CC BY 4.0).

\begin{abstract}
"Water-in-salt" electrolytes with excellent electrochemical and physical properties have been extensively investigated. However, the structural understanding of the lithium bis(trifluoromethane sulfonyl)imide (LiTFSI) in water is still lacking. Here, we perform synchrotron X-ray scattering to systemically study the structural variation of TFSI anions in an aqueous solution under a variety of concentrations and temperatures. There are two different solvation structures in the solution: TFSI $^{-}$solvated structure and TFSI ${ }^{-}$network. As the concentration increases, the TFSI $^{-}$solvated structure gradually disappears while the TFSI $^{-}$network gradually forms. Even at relatively low concentrations, the TFSI' network can be observed. Our experimental results show that these two structures can coexist at a particular concentration, and temperature changes will lead to one structure's formation or disappearance. Also, the TFSI ${ }^{-}$network is the key to obtain a stable electrochemical window under relatively high temperatures.
\end{abstract}

\section{Introduction}

One key component of the Lithium-ion batteries (LIBs) is the electrolyte, which works as an indispensable ion conductor and an electron-insular between the anode and cathode $[1,2]$. Conventional electrolytes usually consist of organic carbonate solvents that are highly volatile and flammable, and $\mathrm{LiPF}_{6}$ salt is chemically unstable [3]. These drawbacks hinder the development of next-generation batteries, requiring higher energy densities, wider operating temperatures, and better safety performance [4]. Compared with the traditional organic electrolytes, nonvolatile and nonflammable aqueous electrolytes could be a promising alternative electrolyte for the next-generation LIBs $[5,6]$. However, the electrochemical stability window of aqueous electrolytes is narrow, and they cannot be used for most of the electrochemical couples in LIBs. Suo et al.'s group [7] reported a new aqueous electrolyte called "water-in-salt" (WIS) containing $21 \mathrm{~m}$ LiTFSI. The WIS electrolyte has a larger electrochemical stability window $(\sim 3.0 \mathrm{~V})$, comparable to the organic electrolytes used in commercial LIBs.
This new finding facilitated the studies on WIS's solvation structure and the transport properties in electrolytes, such as ionic conductivity [8] and transference number [9]. Combining infrared spectroscopy (IR) and molecular dynamics (MD), Lim et al. [8] found that even when the concentration of LiTFSI salt in water is as high as $21 \mathrm{~m}$, there are still bulk-like water molecules acting as a medium for lithium-ion transport. Hydrated lithium ions move through those bulk-like water channels proposed as the lithium-ion conduction mechanism. Borodin et al. [9] investigated the ion solvation and transport in LiTFSI aqueous system with combined MD simulation, small-angle neutron scattering (SANS), and a variety of spectroscopic techniques. They found the disproportionation of cation solvation leads to a heterogeneous liquid structure, and the $\mathrm{Li}^{+}\left(\mathrm{H}_{2} \mathrm{O}\right)_{4}$ domain serves as a percolating channel for fast $\mathrm{Li}^{+}$transports with a high lithium-transference number. With FTIR and MD simulation, Lewis et al. [10] revealed that Li cations contact with TFSI anions directly in the form of contact ion pairs that have a significant population at relatively high concentrations (larger than $3 \mathrm{~m}$ ). Simultaneously, many anions are contacted 
directly in the interconnected ion network mediated by the Li cations.

However, the techniques employed in previous studies, such as NMR [11], Raman [12], and IR spectroscopy [13], are sensitive to the local molecular bonding and orientations and are a challenge to capture the whole structure of the forming clusters and aggregates. Small-angle X-ray scattering (SAXS) provides information about molecules' size and shape and assemblies from 1 to $150 \mathrm{~nm}$ in solution [14-17]. Recently, we investigated the solvation structure and dynamics of highly concentrated LiTFSI aqueous electrolyte combining SAXS with MD simulations [18]. We discovered heterogeneous structure features in $20 \mathrm{~m}$ LiTFSI aqueous solution. Because of this heterogeneous structure, the small number of anions present in the water domain causes a weak overall connection between ions. Such a weak connection promises the movement of $\mathrm{Li}^{+}$in the water domain which is relatively free and results in high correlated transference numbers despite the high viscosity. Although many recent reports have investigated the structures of highly concentrated LiTFSI aqueous electrolytes, the solvation behaviors at different concentrations and temperatures are not entirely understood.

In this work, we conducted a SAXS study to investigate the solvation behavior of LiTFSI aqueous solutions in a wide range of concentration regime of 1 to $20 \mathrm{~m}$. Also, the stability of these samples under different temperatures was studied by in situ SAXS measurements. TFSI anions can form two different structures, $\mathrm{TFSI}^{-}$solvated structure and $\mathrm{TFSI}^{-}$network. At low concentrations, the TFSI solvated structure is the main solvated structure. TFSI ${ }^{-}$solvated structure and TFSI ${ }^{-}$ network coexist as the concentration increases to the medium concentration. At high concentrations, only the TFSI $^{-}$network remains. Also, we found that increasing temperature may promote the conversion between bulk water and interfacial water. The structural changes caused by the temperature are the reversible process. The electrochemical performance of different concentration LiTFSI aqueous solutions under different temperatures was also investigated. The temperature effect was more evident in the oxygen evolution reaction than that in the hydrogen evolution reaction. High concentration of LiTFSI solution is stable at high temperatures.

\section{Materials and Methods}

2.1. Sample Preparation. The electrolytes were prepared by dissolving the lithium bis(trifluoromethane sulfonyl)imide (LiTFSI, >99\%, Sigma-Aldrich), and sodium bis(trifluoromethane sulfonyl)imide (NaTFSI, $>97 \%$, Sigma-Aldrich) in high purity water which conductivity is $18.2 \mathrm{M} \Omega \times \mathrm{cm}$ at $25^{\circ} \mathrm{C}$. All the electrolytes were prepared by molality (molesalt in kg-solvent) used by abbreviated concentrations ( $1 \mathrm{~m}$, $2 \mathrm{~m}, 3 \mathrm{~m}, 5 \mathrm{~m}, 10 \mathrm{~m}, 15 \mathrm{~m}$, and $20 \mathrm{~m}$ ).

2.2. Small-Angle X-Ray Scattering. SAXS experiments were performed at the Advanced Photon Source (APS) 12ID-B and C station of Argonne National Laboratory. The 2D SAXS data were collected on a Pilatus $2 \mathrm{M}$ area detector (DECTRIS
TABle 1: Setups of the MD systems studied in this work.

\begin{tabular}{lccc}
\hline $\begin{array}{l}\text { Nominal } \\
\text { concentration } \\
\left({ }^{*} m\right)\end{array}$ & $\begin{array}{c}\text { MD } \\
\text { concentration } \\
\left({ }^{*} m\right)\end{array}$ & $\begin{array}{c}\text { LiTFSI } \\
\text { number }\end{array}$ & $\begin{array}{c}\text { Water } \\
\text { number }\end{array}$ \\
\hline 1 & 0.99 & 100 & 5600 \\
5 & 5.05 & 500 & 5500 \\
20 & 19.82 & 2000 & 5600 \\
\hline
\end{tabular}

${ }^{*} m=\mathrm{mol} / \mathrm{kg}$.

Ltd.) and incident energy of $12 \mathrm{keV}$. The two-dimensional scattering images were radially averaged over all orientations to produce plots of scattered intensity $I(q)$ versus scattering vector $q$, where $q=4 \pi \sin \theta / \lambda$. The scattering vector, $q$, was calibrated using silver behenate. The samples were loaded into $1.5 \mathrm{~mm}$ diameter quartz capillary tubes and sealed with epoxy for the SAXS measurement. To investigate the LiTFSI and NaTFSI aqueous solution structures under different temperatures, SAXS was investigated in situ. The samples were put in a multisample heater for capillary, which was designed by 12ID-B and C station of Argonne National Laboratory, and the gap between each capillary was $10 \mathrm{~mm}$. This heater was set to change the sample temperature at $10^{\circ} \mathrm{C} / \mathrm{min}$.

2.3. Electrochemical Measurements. Cyclic voltammetry (CV) was carried out using $\mathrm{CHI} 600 \mathrm{E}$ electrochemical working station at a scanning rate of $10 \mathrm{mV} / \mathrm{s}$. 316 stainless steel grid (200-mesh sieve), as working and counter electrodes, was cleaned ultrasonically in alcohol and then washed with high purity water several times and dried before measurement. The electrolytic cell was heated in a water bath, and a thermometer is used to measure the temperature.

2.4. MD Simulation Systems and Methods. MD simulations were performed on 1, 5, and $20 \mathrm{~m}$ LiTFSI aqueous solutions to investigate the transition of the solvation structure of TFSI ${ }^{-}$ion. The details of the MD system setups are listed in Table 1. The initial configurations of the MD simulations were packed using the PACKMOL code [19]. The simulation box is periodic in all three directions. The force fields of $\mathrm{Li}^{+}$ and TFSI $^{-}$ion were taken from some recent works [20]. The TIP3P model was employed for the water molecules [21]. MD simulations were performed using the GROMACS package [22]. The simulations were first equilibrated under the NPT ensemble to stabilize the system density and energy for $30 \mathrm{~ns}$. Then, a $100 \mathrm{~ns}$ trajectory was generated for each simulation under the NVT ensemble [23]. The last $50 \mathrm{~ns}$ trajectory was used for the related analysis. More simulation details were archived in our recent publications.

\section{Results}

3.1. The Evolution of Electrolyte Structure with Varying LiTFSI Concentration. It should be noted that $\mathrm{Li}^{+}$cannot be observed directly through SAXS measurement due to its small scattering factor [15]. Therefore, all information 


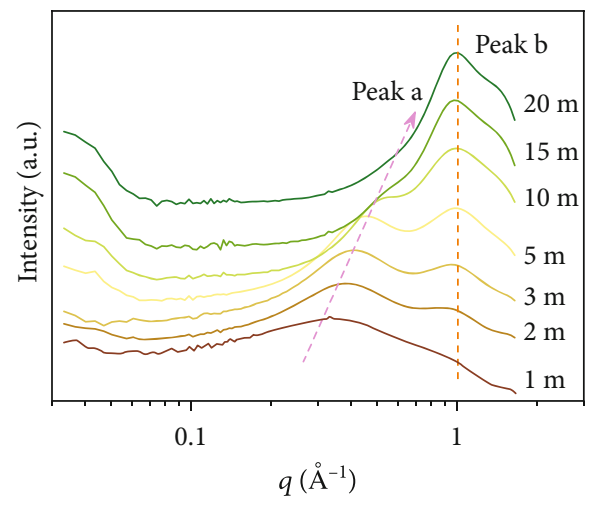

(a)

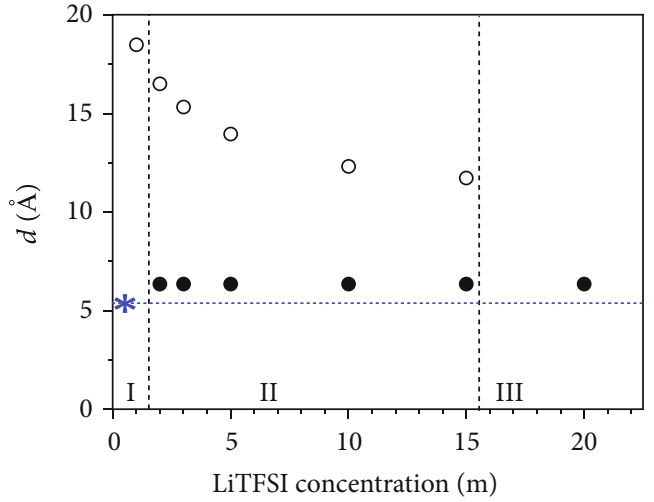

(b)

o Peak a

- Peak b

(I)

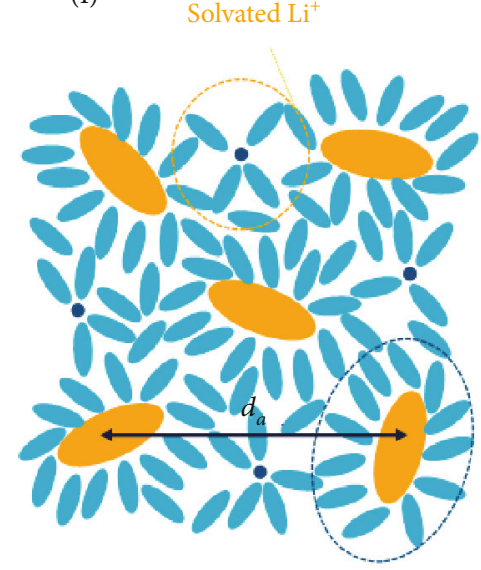

Solvated TFSI ${ }^{-}$
(II)

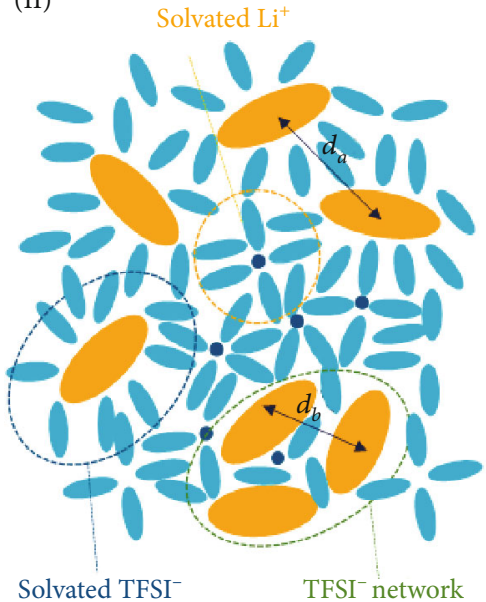

(III) Solvated $\mathrm{Li}^{+}$

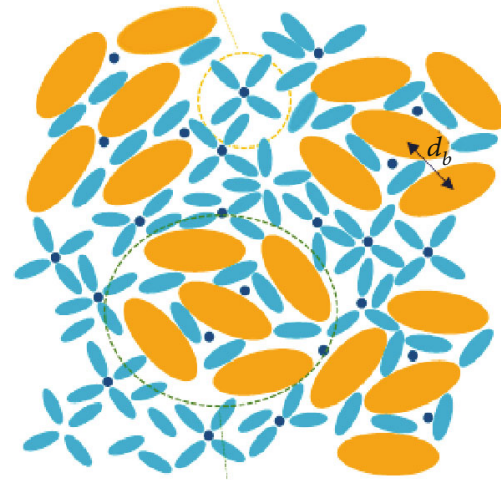

$\mathrm{TFSI}^{-}$network

FIGURE 1: (a) SAXS profile of LiTFSI aqueous electrolytes at different concentrations under room temperature. The data is offset for clarity. (b) $d$ spacing is plotted as a function of LiTFSI concentrations at room temperature. $d$ spacing is calculated by $d=2 \pi / q$. Three concentration regimes are divided: (I) low concentration, (II) medium concentration, and (III) high concentration. $*$ is the $d$ spacing of pure TFSI-LiTFSI [24].

obtained from SAXS measurement is related to the structure of the large TFSI- To fully understand the solvation structure, we systemically changed the LiTFSI concentrations and measured them with SAXS. The SAXS profiles of different LiTFSI concentrations at room temperature are shown in Figure 1(a). We observe two peaks: one is marked as Peak a in the low $q$ part, and the other is labeled as Peak b in the vicinity of $0.99 \AA^{-1}$. As the concentration gradually increases, Peak a position shifts to a higher $q$, which indicates the average distance or $d$ spacing between TFSI ions becomes shorter. Also, the intensity of this peak decreases with increasing concentration. At a high concentration of $20 \mathrm{~m}$, the peak disappears. However, Peak $\mathrm{b}$ begins to appear at a relatively low concentration of $2 \mathrm{~m}$ and its position shifts slightly to a higher $q$ below $10 \mathrm{~m}$ and then remains unchanged as the concentrations increase to a high concentration of $20 \mathrm{~m}$. This suggests that the average $d$ spacing between TFSI $^{-}$networks becomes smaller, and then, there is no change.

The $d$ spacing of the two peaks can be calculated using $d=2 \pi / q$, and its relationship with concentrations is shown in Figure 1(b). There are three concentration regimes, (I) low concentration regime, (II) medium concentration regime, and (III) high concentration regime. At regime I $(<2 \mathrm{~m})$, only Peak a can be observed and $d$ spacing is around $18.5 \AA$. At regime II $(2 \mathrm{~m} \sim 15 \mathrm{~m})$, Peak a and Peak $\mathrm{b}$ coexist. The $d$ spacing of Peak a gradually decreases from 16.2 to $12.0 \AA$, while the $d$ spacing of Peak $\mathrm{b}$ remains at $6.4 \AA$. At regime III $(>15 \mathrm{~m})$, only Peak b can be observed. The two peaks represent two different TFSI $^{-}$solvation structures.

Initially, we assigned Peak b to the charge-ordering of LiTFSI (TFSI ${ }^{-}$and $\mathrm{Li}^{+}$), which is widely used for ionic liquid and molten salt [13]. However, we found Peak b position for NaTFSI solution is the same as LiTFSI (in the later section), which means the cation does not play an essential role for the Peak b. Otherwise, it should be shifted to a lower $q$, considering that the radius of $\mathrm{Na}^{+}$is larger than that of $\mathrm{Li}^{+}$. This result indicates that other $\mathrm{TFSI}^{-}$structures may contribute to this peak. Other possible structures are as follows: (1) $\mathrm{TFSI}^{-}$ and $\mathrm{TFSI}^{-}$are directly in contact, and (2) $\mathrm{TFSI}^{-}$is connected to water molecules (i.e., $\mathrm{TFSI}^{-} \cdots \mathrm{HOH} \cdots \mathrm{TFSI}^{-}$). The $d$ spacing 


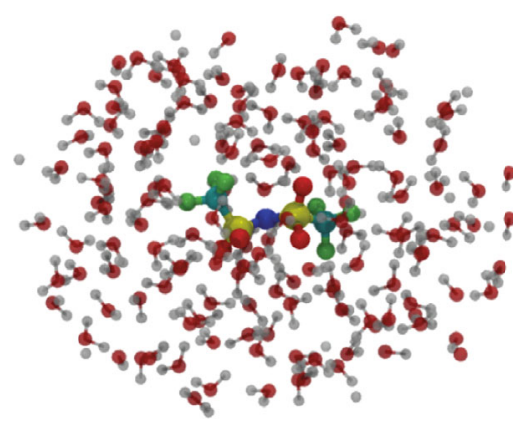

(a)

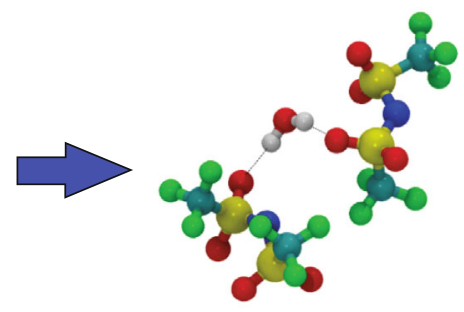

(b)

FIGURE 2: Representative configuration of (a) TFSI ${ }^{-}$solvated structure and (b) TFSI network extracted from $1 \mathrm{~m}$ and $20 \mathrm{~m} \mathrm{MD}$ simulation trajectories. The red, white, yellow, blue, cyan, and green balls denote $\mathrm{O}, \mathrm{H}, \mathrm{S}, \mathrm{N}, \mathrm{C}$, and $\mathrm{F}$ atoms.

between TFSI- network is $6.4 \AA$, which is larger than that of the pure TFSI-Li-TFSI distance [14], as shown in Figure $1(\mathrm{~b})$ ( $*$ is the $d$ spacing of pure LiTFSI obtained from reference, which is $5.3 \AA$ [24]). Therefore, we attribute the formation of this peak to the TFSI ${ }^{-}$network formed by the bridging of water molecules.

As shown in Figure 1(b), the $d$ spacing of Peak a is much larger than pure TFSI-TFSI distance and TFSI networks, indicating the solvation structures are different. We named this solvation structure as the TFSI- solvated structure. Interestingly, we did not observe Peak a when we dissolved LiTFSI in different concentrations of organic solvents (including acetonitrile and propylene carbonate) (Figure S1). The reason is that the water molecules are bipolar while other solvents are not. In an aqueous solution, both cations and anions can be well solvated by water. However, in organic solvents we studied, only cations can be solvated well while anions remain unsolvated and remain relatively free [25-27].

Unlike only one peak is observed at low $(1 \mathrm{~m})$ and high concentration $(20 \mathrm{~m})$, we can observe both Peak a and Peak $\mathrm{b}$ at medium concentrations $(2 \mathrm{~m} \sim 15 \mathrm{~m})$, indicating both the TFSI $^{-}$solvated structure and TFSI $^{-}$network coexist. As the concentration of LiTFSI increases, more TFSI anions will extract free water molecules from the outermost layer, resulting in a shorter distance between TFSI ${ }^{-}$. As a result, Peak a starts to move to a higher $q$. Also, as the concentration increases, TFSI anions begin to form TFSI ${ }^{-}$networks leading to the appearance of Peak $b$, and the intensity increases because of the increased number of TFSI anions in the TFSI ${ }^{-}$ networks. The number of $\mathrm{TFSI}^{-}$solvated structure becomes less, resulting in a decrease in the intensity and disappearance of Peak a. Eventually, only Peak b exists at high concentrations, which indicates that only the TFSI ${ }^{-}$network exists.

The driving forces for the formation of these two different solvated structures are further investigated. Combining our current SAXS work and Lim et al.'s [8] previous IR work, we believe that these two solvated structures are driven by two different hydrogen bonds caused by two different types of water. They have demonstrated two different kinds of water in LiTFSI aqueous solutions: one is bulk water that has hydrogen bonding with each other, and the other is interfacial water, which can form another hydrogen bonding with TFSI ions. From IR spectra, it can be seen that as the LiTFSI concentration gradually increases from 1 to $21 \mathrm{~m}$, the inten- sity of the bulk water peak decreases and the intensity of interfacial water increases. Meanwhile, the bulk water peak has a significantly blue shift, while the interfacial water peak position remains almost unchanged. These results are consistent with our SAXS study. As the concentration increases, Peak a shifts to a high $q$ until it entirely disappears, while the position of Peak $b$ remains unchanged. Therefore, we believe that the TFSI $^{-}$solvated structure is due to the solvation of bulk water, while the formation of TFSI ${ }^{-}$networks is due to the interfacial water and TFSI'. These two structures have been captured with MD simulation, which is shown in Figures 2(a) and 2(b). The concept of interfacial water and bulk water has been well studied in the protein/water system, where the interfacial water forms a strong hydrogen bonding with protein [28].

3.2. Effect of Changing Temperature. TFSI ${ }^{-}$solvated structures are mainly caused by the hydrogen bonding between the bulk water, and TFSI ${ }^{-}$networks are formed through the hydrogen bonding between the $\mathrm{TFSI}^{-}$and the interfacial water. Therefore, we should be able to observe the change in these two structures if the solution is exposed to external stimuli such as temperature because it has been reported that the hydrogen bonding between the bulk water molecules can be destroyed with increasing temperature [29]. To verify this hypothesis, we conduct in situ SAXS studies of LiTFSI at different concentrations and temperatures in aqueous solutions. At low, medium, and high concentrations, we observe three different results. At high concentration, Peak b remains unchanged as temperature varies (Figure 3(a)), indicating that the $d$ spacing has no change (Figure 3(b)).

However, at medium concentrations of $5 \mathrm{~m}$, we observe a different phenomenon, as shown in Figure 3(c). As the temperature gradually increases, Peak a shifts to high $q$, and the intensity decreases, and Peak b shifts to low $q$ with an increase in intensity. The $d$ spacing from both structures is shown in Figure 3(d). Increasing the temperature will break the hydrogen bonding of bulk water molecules. These new water molecules may move into three-dimension TFSI $^{-}$networks and to reorganize with the existing interfacial water to form a new type of interfacial water, which causes Peak b to move to low $q$ and increase in intensity. Conversely, because of the bulk water the solvated TFSI ${ }^{-}$structures becomes less, Peak a shifts to a high $q$ and the intensity 


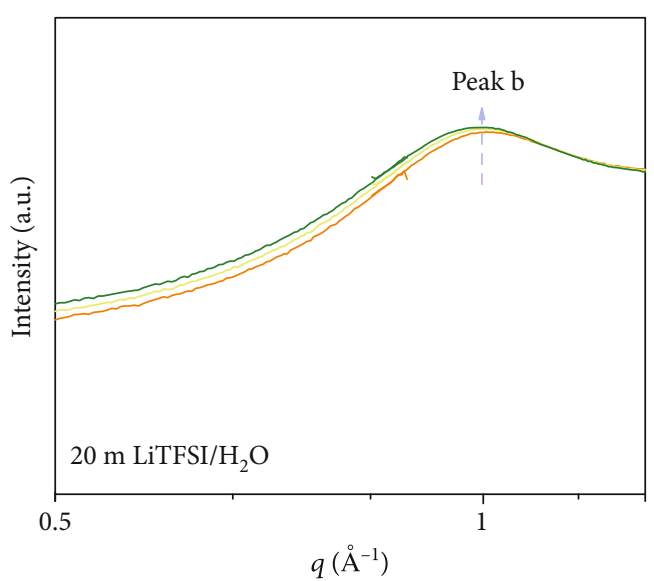

(a)

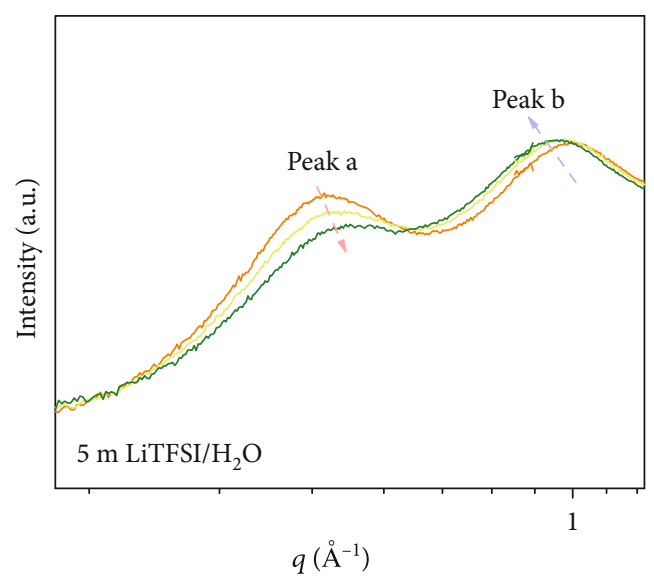

(c)

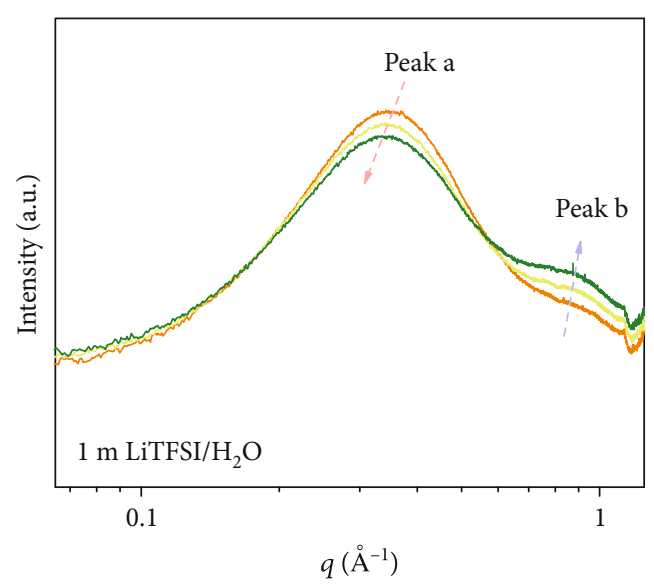

(e)

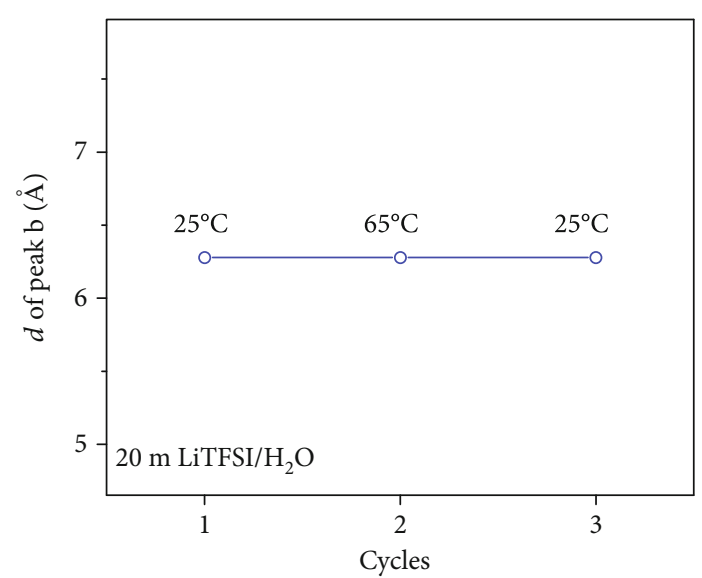

(b)

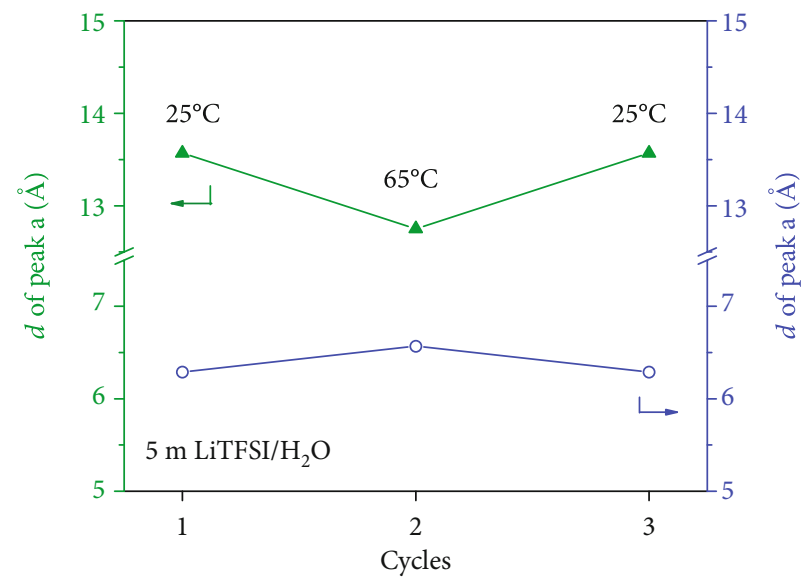

(d)

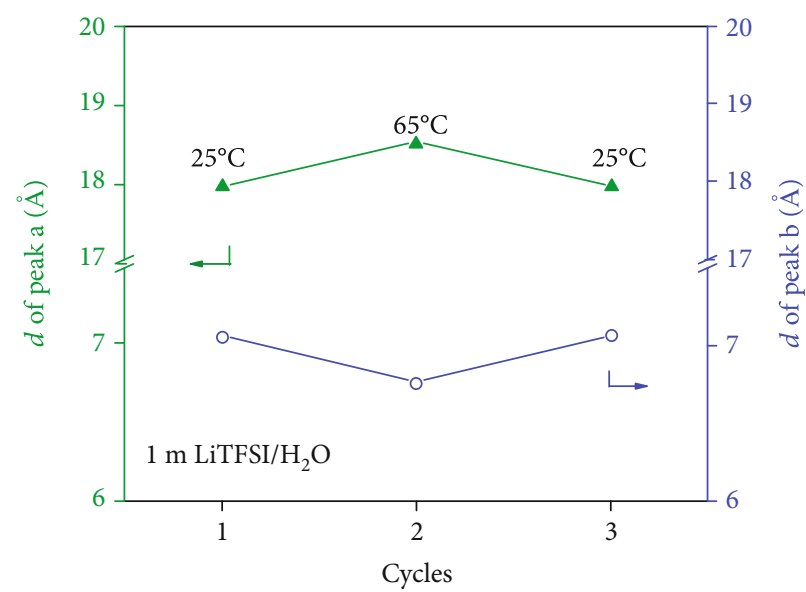

(f)

$25^{\circ} \mathrm{C}$
$-\quad 45^{\circ} \mathrm{C}$
$-\quad 65^{\circ} \mathrm{C}$

FIgURE 3: SAXS profile for the (a) $20 \mathrm{~m}$, (c) $5 \mathrm{~m}$, and (e) $1 \mathrm{~m}$ LiTFSI aqueous solutions at different temperatures. $d$ spacing for the LiTFSI aqueous solutions with different concentrations at various temperatures shown in (b), (d), and (f) during heating/cooling cycles.

decreases. We found the same trend for another medium concentration of $10 \mathrm{~m}$, as shown in Figure S2.

At low concentration $(1 \mathrm{~m})$, the SAXS profiles from 25 to $65^{\circ} \mathrm{C}$ are presented in Figure 3(e), and two peaks can be observed. This means that even at relatively low concentrations, the TFSI ${ }^{-}$networks could form through hydrogen bonding between interfacial water and TFSI'. As the temperature gradually increases, the intensity of Peak a decreases while 


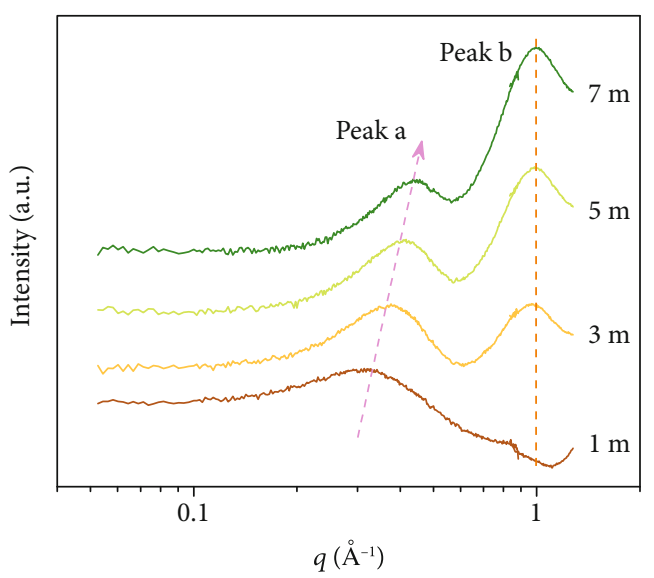

(a)

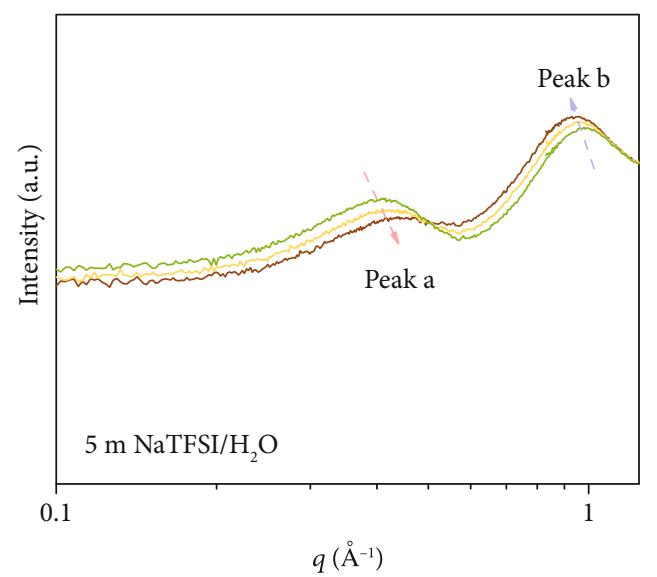

(c)

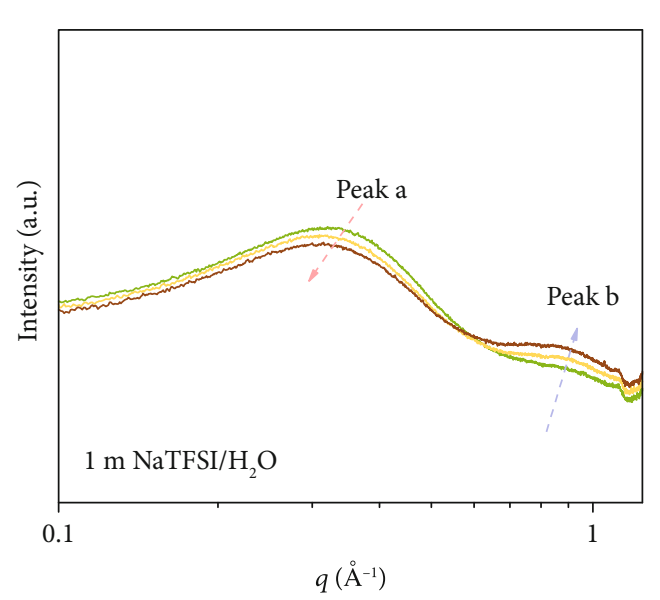

(e)

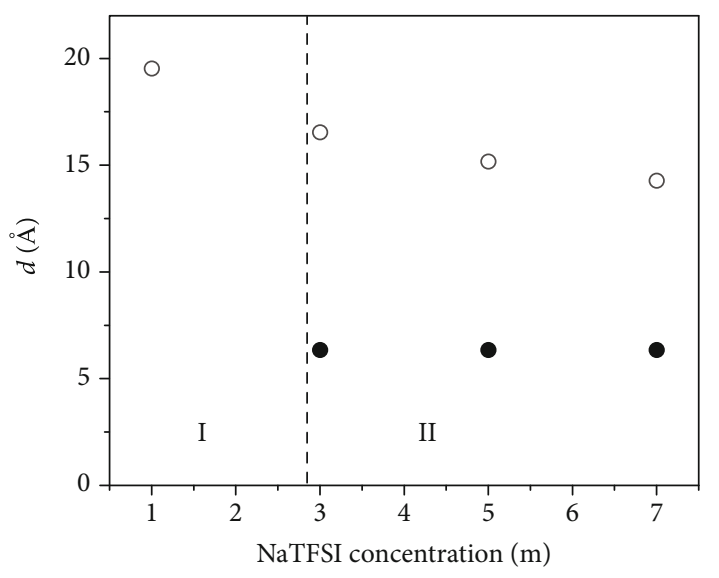

(b)

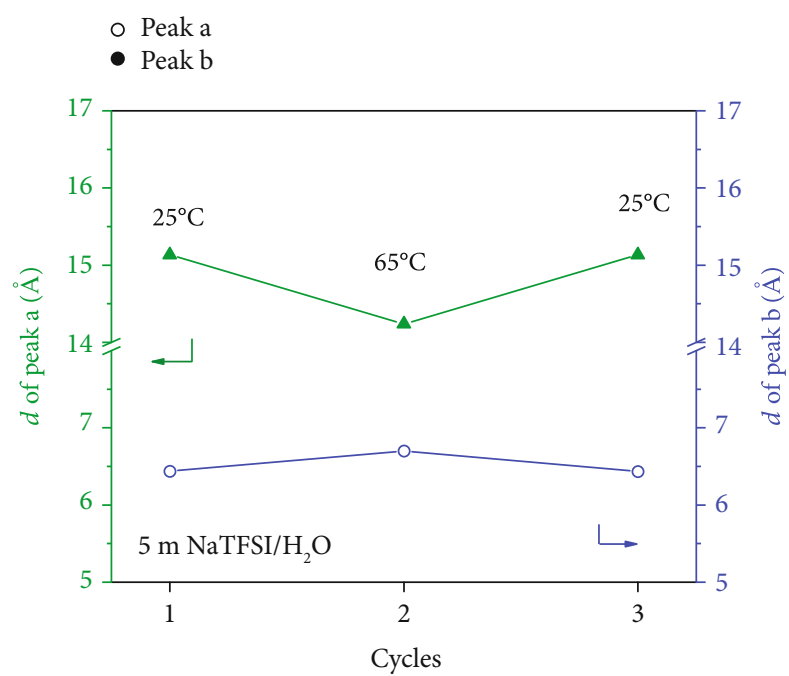

(d)

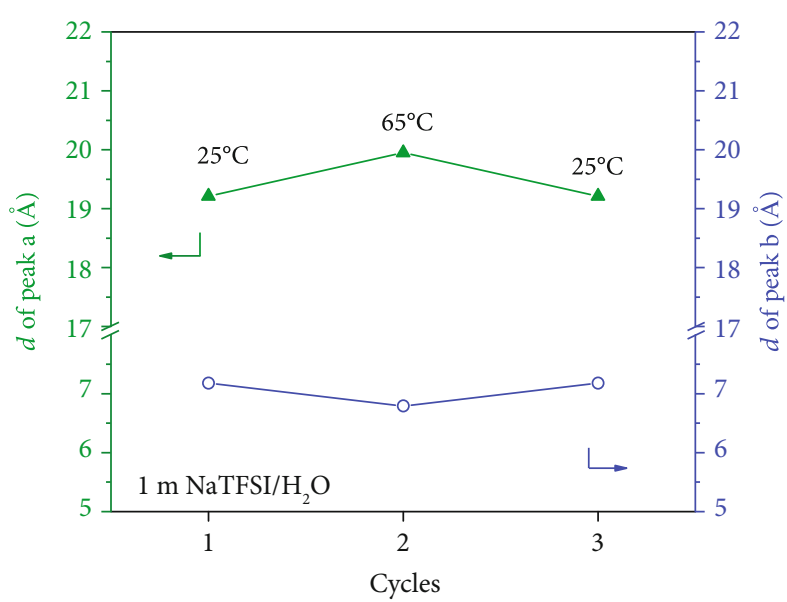

(f)

$$
\begin{array}{r}
-\quad 25^{\circ} \mathrm{C} \\
-\quad 45^{\circ} \mathrm{C} \\
-\quad 65^{\circ} \mathrm{C}
\end{array}
$$

FIGURE 4: (a) SAXS profile for the NaTFSI aqueous electrolytes at different concentrations under room temperature and for clarity the profile offset. (b) $d$ spacing from the SAXS profile for the NaTFSI aqueous electrolytes at different concentrations under room temperature. $d$ spacing is calculated by $d=2 \pi / q$. Two regimes can be divided: (I) low concentration and (II) medium concentration. SAXS profile for the (c) $5 \mathrm{~m}$ and (d) $1 \mathrm{~m}$ NaTFSI aqueous electrolytes at different temperatures. $d$ spacing for the NaTFSI aqueous solutions with different concentrations at various temperatures shown in $(\mathrm{d}, \mathrm{f})$ during heating/cooling cycles. 


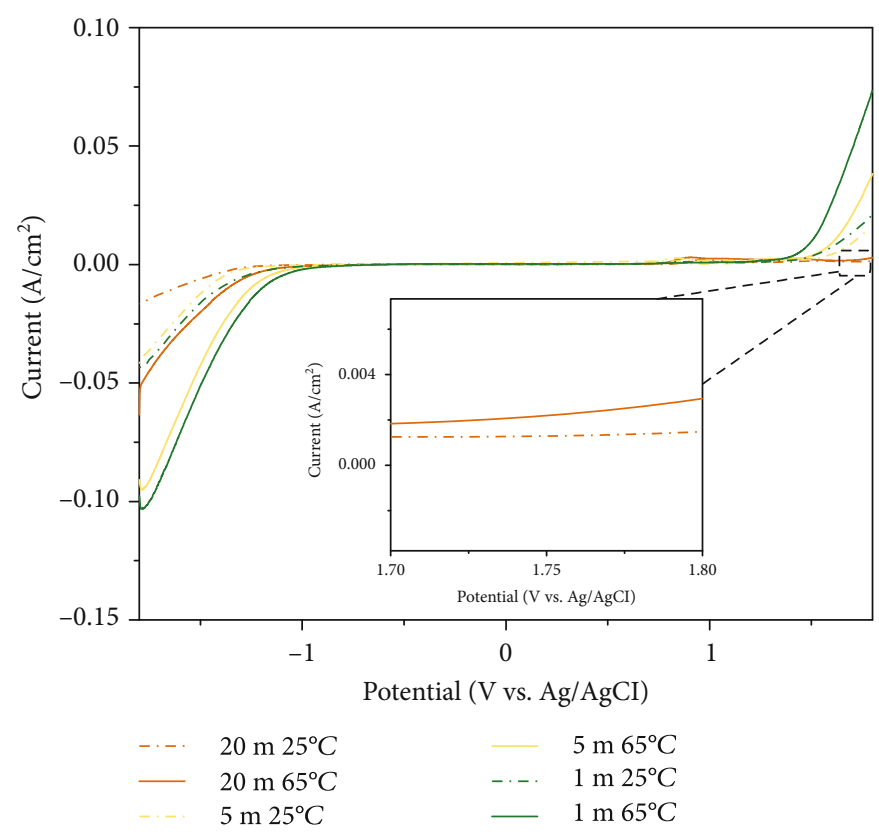

Figure 5: The electrochemical stability window of $1 \mathrm{~m}, 5 \mathrm{~m}$, and $20 \mathrm{~m}$ aqueous electrolytes on nonactive electrolytes under different temperatures.

the intensity of Peak b increases, which is the result of the relative population of the two TFSI ${ }^{-}$species gradually changing with increasing temperature. Heating will break the hydrogen bonding from bulk water and may promote the formation of new interfacial hydrogen bonding. As a result, the TFSI ${ }^{-}$solvated structures become less and more TFSI $^{-}$interacts with interfacial water to form TFSI $^{-}$network. The reduced number of solvated TFSI ${ }^{-}$causes Peak a shifts slightly to the low $q$ [28], and the $d$ value is shown in Figure 3(f).

It should be noted that this temperature effect is entirely reversible regardless of the concentration of LiTFSI. As shown in Figures 3(b), 3(d), and 3(f), the value of the LiTFSI aqueous solutions under different temperatures is reversible. For example, at a medium concentration of $5 \mathrm{~m}$ as shown in Figure 3(d), the $d$ values of Peak a and Peak b are $13.6 \AA$ and $6.3 \AA$ at $25^{\circ} \mathrm{C}$, respectively, and they change to $12.8 \AA$ and $6.6 \AA$ as the temperature rises to $65^{\circ} \mathrm{C}$. When the temperature is lowered to $25^{\circ} \mathrm{C}$, the $d$ value returns to $13.6 \AA$ and $6.3 \AA$. We can observe the same phenomenon for the low $(1 \mathrm{~m})$, medium (10 m) (Figure S2), and high concentration (20 m) LiTFSI aqueous solutions.

Next, we tested NaTFSI in an aqueous solution and found similar phenomena as LiTFSI aqueous solutions. From Figure 4(a), we can observe the two peaks coexist. As the concentration increases, Peak a moves to high $q$ and its intensity decreases while Peak $\mathrm{b}$ appears at a concentration of $3 \mathrm{~m}$ and its position $\left(q=0.99 \AA^{-1}\right)$ remains almost no change. Unlike LiTFSI solution, it only shows low and medium concentration regimes (Figure 4(b)) because of the lower solubility of NaTFSI. At a medium concentration of $5 \mathrm{~m}$ (Figure 4(c)), Peak a shifts to higher $q$ with a decreased intensity and Peak b shifts to lower $q$ with an increase in intensity as temperature increases. A similar trend is observed for other medium concentrations of $3 \mathrm{~m}$ and $7 \mathrm{~m}$ (Figure S3 and S4). For the low concentration NaTFSI aqueous electrolyte $(1 \mathrm{~m})$, as shown in Figure 4(e), the intensity of Peak a decreases while the intensity of Peak b increases. We also can observe that Peak a shifts to a lower $q$ with the temperature increasing, which is the same as $1 \mathrm{~m}$ LiTFSI aqueous solution. This temperature effect is also completely reversible regardless of the concentration of NaTFSI. As shown in Figures 4(d) and $4(\mathrm{f})$, the variation of the $d$ value of the NaTFSI aqueous solutions under different temperatures is reversible. Based on the SAXS profiles of LiTFSI and NaTFSI aqueous solutions, we find that TFSI $^{-}$salts with cations in the same group have the same behavior as a function of concentration and temperature.

\subsection{Electrochemical Stability Test under Different} Temperatures. From SAXS results at different temperatures, we can observe the structural changes of LiTFSI aqueous solutions at different concentrations. We believe that this structural change may lead to different electrochemical performances. Therefore, we investigated the electrochemical stability window of low $(1 \mathrm{~m})$, medium $(5 \mathrm{~m})$, and high $(20 \mathrm{~m})$ LiTFSI aqueous electrolytes under different temperatures, as shown in Figure 5. The electrochemical window was evaluated on the stainless steel electrode by cyclic voltammetry (CV). Compared with $1 \mathrm{~m}$ LiTFSI aqueous electrolyte, the overall stability window of $20 \mathrm{~m}$ LiTFSI aqueous electrolytes has been expanded to ca. 3.0 V. The onset potential of the hydrogen evolution reaction (HER) and oxygen evolution reaction (OER) shifts to more negative and positive values, respectively. The phenomenon is consistent with previous finding by Suo et al. [7]. With increasing the temperature, the electrochemical stability windows for $1 \mathrm{~m}, 5 \mathrm{~m}$, and $20 \mathrm{~m}$ LiTFSI aqueous electrolytes were narrow. Since the positively charged electrode in the OER will contact an excess of 
TFSI anions, the temperature effect will be more pronounced on the anode side. We can observe from Figure 5 that the change of current on the OER of the $1 \mathrm{~m}$ LiTFSI aqueous electrolytes is more significant than that of $5 \mathrm{~m}$ and $20 \mathrm{~m}$ LiTFSI solutions. For a $20 \mathrm{~m}$ LiTFSI aqueous electrolyte, the current changes from $0.002 \mathrm{~A} / \mathrm{cm}^{2}$ to $0.003 \mathrm{~A} / \mathrm{cm}^{2}$ under $1.8 \mathrm{~V}$ as the temperature increases from $25^{\circ} \mathrm{C}$ to $65^{\circ} \mathrm{C}$, but for $1 \mathrm{~m} \mathrm{LiTFSI}$ aqueous electrolyte, the current changes from $0.02 \mathrm{~A} / \mathrm{cm}^{2}$ to $0.07 \mathrm{~A} / \mathrm{cm}^{2}$. The rate of change of $1 \mathrm{~m}$ LiTFSI aqueous electrolytes is 2.5 times that of $20 \mathrm{~m}$ LiTFSI aqueous electrolytes (the current changes from $0.02 \mathrm{~A} / \mathrm{cm}^{2}$ to $0.04 \mathrm{~A} / \mathrm{cm}^{2}$ ) and 5 times of $20 \mathrm{~m} \mathrm{LiTFSI} \mathrm{aque-}$ ous electrolytes (Table S1). This phenomenon can be ascribed to the gradual formation of hydrogen bonding between water molecules and TFSI anions with the concentration increases.

Water molecules forming the hydration sphere of lithium ions present at the electrode surface can be stripped off and reduced to form hydrogen. From Table S1, the current change rates of $1 \mathrm{~m}, 5 \mathrm{~m}, 10 \mathrm{~m}, 15 \mathrm{~m}$, and $20 \mathrm{~m}$ LiTFSI aqueous solutions under $-1.8 \mathrm{~V}$ HER onset potential are almost the same (150\%), which means there are many free water molecules which solvated $\mathrm{Li}^{+}$in the high concentration LiTFSI aqueous electrolyte at the cathode electrode. A schematic presentation of the species presents negatively and positively charged electrodes in low and high concentration shown in Figure S5.

\section{Discussion}

In summary, we studied LiTFSI solutions with synchrotron $\mathrm{X}$-ray scattering technique by systemically varying the LiTFSI concentration and temperature. According to SAXS results, LiTFSI aqueous solutions can be divided into three concentration regimes: low concentration, medium concentration, and high concentration. At a low concentration solution regime, only $\mathrm{TFSI}^{-}$solvated structures exist at room temperature. However, the TFSI ${ }^{-}$networks can be formed with increasing temperature. For a medium concentration solution regime, two structures (TFSI ${ }^{-}$solvated structure and TFSI $^{-}$network) coexist. There is only one structure at room temperature at a high concentration solution regime, TFSI $^{-}$network, where all TFSI ${ }^{-}$have been involved in forming TFSI $^{-}$networks. TFSI $^{-}$network structure at high temperature even improves the electrochemical high-temperature stability.

\section{Data Availability}

All data needed to evaluate the conclusions in the paper are presented in the paper and/or the Supplementary Materials. Additional data related to this paper may be requested from the authors T. L.

\section{Conflicts of Interest}

The authors declare that there is no conflict of interest regarding the publication of this article.

\section{Acknowledgments}

This work was supported as part of the Joint Center for Energy Storage Research, an Energy Innovation Hub, funded by the U.S. Department of Energy, Office of Science, Basic Energy Sciences. This research used resources of the Advanced Photon Source, a U.S. Department of Energy (DOE) Office of Science User Facility operated for the DOE Office of Science by Argonne National Laboratory under Contract No. AC02-06CH11357.

\section{Supplementary Materials}

Figure S1: SAXS profile for the (a) acetonitrile and (b) propylene carbonate at different concentrations under room temperature. Figure S2: (a) SAXS profile for the $10 \mathrm{~m}$ LiTFSI aqueous solutions at different temperatures. SAXS curves for the $10 \mathrm{~m}$ LiTFSI aqueous solution at various temperatures shown in (b) during heating/cooling cycles. Figure S3: (a) SAXS profile for the $7 \mathrm{~m}$ NaTFSI aqueous solutions at different temperatures. SAXS curves for the $7 \mathrm{~m}$ NaTFSI aqueous solution at various temperatures shown in (b) during heating/cooling cycles. Figure S4: (a) SAXS profile for the $3 \mathrm{~m}$ NaTFSI aqueous solutions at different temperatures. SAXS curves for the $3 \mathrm{~m}$ NaTFSI aqueous solution at various temperatures shown in (b) during heating/cooling cycles. Figure S5: schematic representation of the ionic environment close to the electrode surface under polarization for the low and high concentration LiTFSI aqueous electrolytes. Table S1: current density of $1 \mathrm{~m}, 5 \mathrm{~m}, 10 \mathrm{~m}, 15 \mathrm{~m}$, and $20 \mathrm{~m}$ aqueous electrolytes on nonactive electrolytes under different temperatures. (Supplementary Materials)

\section{References}

[1] M. Armand, F. Endres, D. R. MacFarlane, H. Ohno, and B. Scrosati, "Ionic-liquid materials for the electrochemical challenges of the future," Nature Materials, vol. 8, no. 8, pp. 621-629, 2009.

[2] M. Armand and J. M. Tarascon, "Building better batteries," Nature, vol. 451, no. 7179, pp. 652-657, 2008.

[3] S.-T. Myung, Y. Sasaki, S. Sakurada, Y.-K. Sun, and H. Yashiro, "Electrochemical behavior of current collectors for lithium batteries in non-aqueous alkyl carbonate solution and surface analysis by ToF-SIMS," Electrochimica Acta, vol. 55, no. 1, pp. 288-297, 2009.

[4] D. R. MacFarlane, M. Forsyth, P. C. Howlett et al., "Ionic liquids and their solid-state analogues as materials for energy generation and storage," Nature Reviews Materials, vol. 1, no. 2, pp. 1-15, 2016.

[5] H. Kim, J. Hong, K. Y. Park, H. Kim, S. W. Kim, and K. Kang, "Aqueous rechargeable $\mathrm{Li}$ and $\mathrm{Na}$ ion batteries," Chemical Reviews, vol. 114, no. 23, pp. 11788-11827, 2014.

[6] Y. Wang, J. Yi, and Y. Xia, "Recent progress in aqueous lithium-ion batteries," Advanced Energy Materials, vol. 2, no. 7, pp. 830-840, 2012.

[7] L. Suo, O. Borodin, T. Gao et al., "'Water-in-salt" electrolyte enables high-voltage aqueous lithium-ion chemistries," Science, vol. 350, no. 6263, pp. 938-943, 2015. 
[8] J. Lim, K. Park, H. Lee, J. Kim, K. Kwak, and M. Cho, "Nanometric water channels in water-in-salt lithium ion battery electrolyte," Journal of the American Chemical Society, vol. 140, no. 46, pp. 15661-15667, 2018.

[9] O. Borodin, L. Suo, M. Gobet et al., "Liquid structure with nano-heterogeneity promotes cationic transport in concentrated electrolytes," ACS Nano, vol. 11, no. 10, pp. 1046210471, 2017.

[10] N. H. C. Lewis, Y. Zhang, B. Dereka, E. V. Carino, E. J. Maginn, and A. Tokmakoff, "Signatures of ion pairing and aggregation in the vibrational spectroscopy of super-concentrated aqueous lithium bistriflimide solutions," The Journal of Physical Chemistry C, vol. 124, no. 6, pp. 3470-3481, 2020.

[11] A. D. Pauric, I. C. Halalay, and G. R. Goward, "Combined NMR and molecular dynamics modeling study of transport properties in sulfonamide based deep eutectic lithium electrolytes: LiTFSI based binary systems," Physical Chemistry Chemical Physics, vol. 18, no. 9, pp. 6657-6667, 2016.

[12] Q. Dou, S. Lei, D.-W. Wang et al., "Safe and high-rate supercapacitors based on an "acetonitrile/water in salt" hybrid electrolyte," Energy \& Environmental Science, vol. 11, no. 11, pp. 3212-3219, 2018.

[13] D. M. Seo, P. D. Boyle, R. D. Sommer, J. S. Daubert, O. Borodin, and W. A. Henderson, "Solvate structures and spectroscopic characterization of LiTFSI electrolytes," The Journal of Physical Chemistry B, vol. 118, no. 47, pp. 1360113608, 2014.

[14] T. Li, A. J. Senesi, and B. Lee, "Small angle X-ray scattering for nanoparticle research," Chemical Reviews, vol. 116, no. 18, pp. 11128-11180, 2016.

[15] Z. Feng, E. Sarnello, T. Li, and L. Cheng, "Communication-microscopic view of the ethylene carbonate based lithium-ion battery electrolyte by X-ray scattering," Journal of the Electrochemical Society, vol. 166, no. 2, pp. A47-A49, 2019.

[16] C. D. Putnam, M. Hammel, G. L. Hura, and J. A. Tainer, "X-ray solution scattering (SAXS) combined with crystallography and computation: defining accurate macromolecular structures, conformations and assemblies in solution," Quarterly reviews of biophysics, vol. 40, no. 3, pp. 191-285, 2007.

[17] K. Qian, R. E. Winans, and T. Li, "Insights into the nanostructure, solvation, and dynamics of liquid electrolytes through small-angle X-ray scattering," Advanced Energy Materials, vol. 20281, pp. 1-22, 2020.

[18] Z. Yu, L. A. Curtiss, R. E. Winans, Y. Zhang, T. Li, and L. Cheng, "Asymmetric composition of ionic aggregates and the origin of high correlated transference number in waterin-salt electrolytes," Journal of Physical Chemistry Letters, vol. 11, no. 4, pp. 1276-1281, 2020.

[19] L. Martinez, R. Andrade, E. G. Birgin, and J. M. Martinez, "PACKMOL: a package for building initial configurations for molecular dynamics simulations," Journal of Computational Chemistry, vol. 30, no. 13, pp. 2157-2164, 2009.

[20] B. Doherty, X. Zhong, S. Gathiaka, B. Li, and O. Acevedo, "Revisiting OPLS force field parameters for ionic liquid simulations," Journal of Chemical Theory and Computation, vol. 13, no. 12, pp. 6131-6145, 2017.

[21] K. P. Jensen and W. L. Jorgensen, "Halide, ammonium, and alkali metal ion parameters for modeling aqueous solutions," Journal of Chemical Theory and Computation, vol. 2, no. 6, pp. 1499-1509, 2006.
[22] W. L. Jorgensen, J. Chandrasekhar, J. D. Madura, R. W. Impey, and M. L. Klein, "Comparison of simple potential functions for simulating liquid water," The Journal of Chemical Physics, vol. 79, no. 2, pp. 926-935, 1983.

[23] C. K. Berk Hess, D. van der Spoel, and E. Lindahl, "GROMACS 4: algorithms for highly efficient, load-balanced, and scalable molecular simulation," Journal of Chemical Theory and Computation, vol. 4, no. 3, pp. 435-447, 2008.

[24] K. Shimizu, A. A. Freitas, R. Atkin et al., "Structural and aggregate analyses of (Li salt + glyme) mixtures: the complex nature of solvate ionic liquids," Physical Chemistry Chemical Physics, vol. 17, no. 34, pp. 22321-22335, 2015.

[25] A. von Cresce and K. Xu, "Preferential solvation of $\mathrm{Li}^{+}$directs formation of interphase on graphitic anode," Electrochemical and Solid State Letters, vol. 14, no. 10, 2011.

[26] A. Von Wald Cresce, M. Gobet, O. Borodin et al., "Anion solvation in carbonate-based electrolytes," Journal of Physical Chemistry C, vol. 119, no. 49, pp. 27255-27264, 2015.

[27] K. Xu and A. von Wald Cresce, "Li+-solvation/desolvation dictates interphasial processes on graphitic anode in $\mathrm{Li}$ ion cells," International Journal of Materials Research, vol. 27, no. 18, pp. 2327-2341, 2012.

[28] M. Tarek and D. J. Tobias, "Role of protein-water hydrogen bond dynamics in the protein dynamical transition," Physical Review Letters, vol. 88, no. 13, pp. 1-4, 2002.

[29] C. D. C. Jared, D. Smith, K. R. Wilson, B. M. Messer, R. C. Cohen, and R. J. Saykally, "Energetics of hydrogen bond network rearrangements in liquid water," Science, vol. 306, pp. 851-853, 2016. 